TITLE:

\title{
Multi-Port Model Order Reduction Using a Matrix Cauer Ladder Network
}

\section{$\operatorname{AUTHOR}(\mathrm{S})$ :}

Matsuo, Tetsuji; Fujiwara, Takayuki; Kuriyama, Kenta; Sugahara, Kengo; Kameari, Akihisa; Tokumasu, Tadashi; Shindo, Yuji

\section{CITATION:}

Matsuo, Tetsuji ...[et al]. Multi-Port Model Order Reduction Using a Matrix Cauer Ladder Network. IEEE Transactions on Magnetics 2020, 56(2): 7506905.

\section{ISSUE DATE:}

2020-02

URL:

http://hdl.handle.net/2433/254201

\section{RIGHT:}

(c) 2020 IEEE. Personal use of this material is permitted. Permission from IEEE must be obtained for all other uses, in any current or future media, including reprinting/republishing this material for advertising or promotional purposes, creating new collective works, for resale or redistribution to servers or lists, or reuse of any copyrighted component of this work in other works.; この論文は出版社版でありません。引用の際には出版社版をご確認ご利用ください。;This is not the published version. Please cite only the published version. 


\title{
Multi-port Model Order Reduction Using a Matrix Cauer Ladder Network
}

\author{
Tetsuji Matsuo ${ }^{1}$, Takayuki Fujiwara ${ }^{1}$, Kenta Kuriyama ${ }^{1}$, Kengo Sugahara $^{2}$, Akihisa Kameari ${ }^{3}$, Tadashi Tokumasu ${ }^{4}$ \\ and Yuji Shindo ${ }^{5}$ \\ ${ }^{1}$ Graduate School of Engineering, Kyoto University, Kyoto 615-8510, Japan \\ ${ }^{2}$ Faculty of Science and Engineering, Kindai University, Higashiosaka 577-8502, Japan \\ ${ }^{3}$ Science Solutions International Laboratory, Inc., Tokyo 153-0065, Japan \\ ${ }^{4}$ Toshiba Infrastructure Systems Solutions Corporation, Fuchu, 183-8511, Japan \\ ${ }^{5}$ Kawasaki Heavy Industries, Ltd., Akashi 673-8666, Japan
}

\begin{abstract}
To achieve efficient multi-port model order reduction, a multi-port Cauer ladder network (CLN) method is formulated that directly yields resistance and inductance matrices that consist of the network elements in the matrix Cauer form. The eddy current field driven by multiple power sources is accurately reconstructed using a small number of network elements. The matrix Cauer form achieves faster convergence of the transfer function than a single-port CLN method and almost the same convergence as a block PVL method.
\end{abstract}

Index Terms - Cauer ladder network, finite element eddy current analysis, model order reduction, multiport.

\section{INTRODUCTION}

$\mathrm{C}$ URRENT computer-aided design of electric machines requires the coupled analysis of the electromagnetic field and control circuit. When the electric machine is driven by a power electronic control circuit, the control circuit that operates at a high switching frequency is analyzed simultaneously with the eddy current (EC) field induced in the machine. However, EC analysis for high-frequency ranges requires fine finite element (FE) division because of thin skin depths and small time steps.

For an efficient representation of the EC field, several model order reduction (MOR) methods have been developed, such as the PVL method [1]-[4] and POD method [5], [6]. The CLN method [7], [8] is an energy-based MOR method that provides a clear physical interpretation of the network elements. The CLN method was originally derived as a singleport system similar to other MOR methods. Most electric machines and devices, however, have multiple ports that arise from multiple windings, such as transformers.

To apply control theory with a multiple-input/multipleoutput (MIMO) system to electric machine control, a multiport MOR method [2], [3], [6], [9] is required to yield a MIMO transfer function. The impedance/admittance matrix representation is useful for connecting several subsystems in a blockwise style.

Following the preliminary examination of multi-port CLN in [10], in this paper, we provide a mathematical derivation of multi-port CLN method based on the matrix Cauer form, which is a natural extension of a single-port CLN [8]. Its practical application to three-phase motor analysis is given in [11].

\section{MULTIPORT CLN METHOD}

\section{A. Multiport CLN Procedure}

Vector potential $\boldsymbol{A}$ and electric field $\boldsymbol{E}$ are represented in FE space as

$$
\begin{aligned}
& \boldsymbol{A}=\sum_{i} a_{i} \boldsymbol{w}_{i}^{1}, \quad \boldsymbol{a}=\left[a_{1}, a_{2}, \ldots\right]^{\mathrm{T}} \\
& \boldsymbol{E}=\sum_{i} e_{i} \boldsymbol{w}_{i}^{1}, \boldsymbol{e}=\left[e_{1}, e_{2}, \ldots\right]^{\mathrm{T}},
\end{aligned}
$$

where $a_{i}$ and $e_{i}$ are the line integrals of $\boldsymbol{A}$ and $\boldsymbol{E}$ on edge $i$, and $\boldsymbol{w}_{i}^{1}$ is the edge element.

Using edge-face incident matrix $\boldsymbol{C}$, the governing equation of the EC field is

$$
C^{\mathrm{T}} \boldsymbol{v} \boldsymbol{C a}=\sigma e, C e=-\mathrm{j} \omega \boldsymbol{C a},
$$

where $\boldsymbol{C}^{\mathrm{T}} \boldsymbol{v} \boldsymbol{C}$ is the coefficient matrix for FE EC analysis; the reluctance matrix $\boldsymbol{v}$ and conductivity matrix $\boldsymbol{\sigma}$ are given by

$$
\begin{aligned}
& \boldsymbol{v}=\left\{v_{i j}\right\}, v_{i j}=\int_{\Omega} \frac{1}{\mu} \boldsymbol{w}_{i}^{2} \cdot \boldsymbol{w}_{j}^{2} \mathrm{~d} \Omega \\
& \boldsymbol{\sigma}=\left\{\sigma_{i j}\right\}, \sigma_{i j}=\int_{\Omega} \sigma \boldsymbol{w}_{i}^{1} \cdot \boldsymbol{w}_{j}^{1} \mathrm{~d} \Omega,
\end{aligned}
$$

where $\mu$ is the permeability, $\sigma$ is the conductivity, and $\boldsymbol{w}_{j}^{2}$ is the facial element of face $j$. For simplicity, the A-formulation is discussed in this paper. The A- $\phi$ formulation can be applied to the CLN method [12], which is often required especially in the three-dimensional analysis. The Dirichlet or Neumann condition is assumed at the boundary. It is supposed that the operation of $\left[\boldsymbol{C}^{\mathrm{T}} \mathbf{v} \boldsymbol{C}\right]^{-1}$ is possible. If necessary, a gauge condition is imposed to (2).

Let the number of ports be $M$. In a similar manner to the single-port version [7], [8], a multiport CLN is constructed as 


$$
\begin{aligned}
& \boldsymbol{C}^{\mathrm{T}} \boldsymbol{v} \boldsymbol{C} \tilde{\boldsymbol{a}}_{2 n+1}=\boldsymbol{\sigma} \boldsymbol{e}_{2 n} \boldsymbol{R}_{2 n}, \boldsymbol{a}_{2 n+1}=\boldsymbol{a}_{2 n-1}+\tilde{\boldsymbol{a}}_{2 n+1} \\
& \tilde{\boldsymbol{e}}_{2 n+2}=-\boldsymbol{a}_{2 n+1} \boldsymbol{L}_{2 n+1}{ }^{-1}, \boldsymbol{e}_{2 n+2}=\boldsymbol{e}_{2 n}+\tilde{\boldsymbol{e}}_{2 n+2} \\
& \boldsymbol{R}_{2 n}{ }^{-1}=\boldsymbol{e}_{2 n}^{\mathrm{T}} \boldsymbol{\sigma} \boldsymbol{e}_{2 n}, \boldsymbol{L}_{2 n+1}=\boldsymbol{a}_{2 n+1}^{\mathrm{T}} \boldsymbol{C}^{\mathrm{T}} \boldsymbol{v} \boldsymbol{C} \boldsymbol{a}_{2 n+1}
\end{aligned}
$$

where $\boldsymbol{R}_{2 n}$ and $\boldsymbol{L}_{2 n+1}$ are $M \times M$ resistance and inductance matrices, and $\boldsymbol{e}_{2 n}=\left(\boldsymbol{e}_{1,2 n}, \ldots, \boldsymbol{e}_{M, 2 n}\right)$ and $\boldsymbol{a}_{2 n+1}=\left(\boldsymbol{a}_{1,2 n+1}, \ldots\right.$, $\left.\boldsymbol{a}_{M, 2 n+1}\right)$ are the $n$-th basis vector matrices. The $m$-th columns $\boldsymbol{e}_{m, 2 n}$ and $\boldsymbol{a}_{m, 2 n+1}$ are field basis vectors when source $m$ has a unit power source and the other sources are zero.

Unit power sources are given to start the CLN procedure. For example, if a unit voltage is given to port $m(m=1, \ldots, M)$ as the boundary condition and the resultant scalar potential distribution is represented as $\phi_{m}$, then the initial condition is given by the electrostatic field $\boldsymbol{e}_{0}$ :

$$
\boldsymbol{a}_{-1}=0, \boldsymbol{e}_{0}=-\boldsymbol{G}\left[\boldsymbol{\phi}_{1}, \ldots, \phi_{M}\right],
$$

where $\boldsymbol{G}$ is the node-edge incidence matrix that corresponds to the grad operator, which satisfies $\boldsymbol{C G}=0$. If a unit direct current is given as the power input to port $m$ and it imposes current density $\boldsymbol{j}_{0 m}$, then the initial condition is given by

$$
\boldsymbol{e}_{0}=0, \boldsymbol{a}_{1}=\left(\boldsymbol{C}^{\mathrm{T}} \boldsymbol{v} \boldsymbol{C}\right)^{-1}\left[\boldsymbol{j}_{01}, \ldots, \boldsymbol{j}_{0 M}\right] .
$$

In this case, $\boldsymbol{R}_{0}$ can be skipped. After power sources are given at the first step, the first equation of (5) is solved for $\tilde{\boldsymbol{a}}_{2 n+1}$ with the Dirichlet or Neumann boundary condition without power inputs in the following steps [13].

The first equation of (5) yields $\tilde{\boldsymbol{a}}_{2 n+1}$ as a new set of basis vectors from which the second equation gives $\boldsymbol{a}_{2 n+1}$ so that $\boldsymbol{a}_{2 n+1}$ will be orthogonal to other $\boldsymbol{a}_{2 i+1} \quad(i<n)$. Similar to the single-port version, it can be proven that procedure (5)-(7) generates the basis vectors that have blockwise orthogonality

$$
\boldsymbol{e}_{2 i}^{\mathrm{T}} \boldsymbol{\sigma e}_{2 j}=\delta_{i j} \boldsymbol{R}_{2 i}{ }^{-1}, \boldsymbol{a}_{2 i+1}^{\mathrm{T}} \boldsymbol{C}^{\mathrm{T}} \boldsymbol{v} \boldsymbol{C} \boldsymbol{a}_{2 j+1}=\delta_{i j} \boldsymbol{L}_{2 i+1}
$$

where $\delta_{i j}$ is the Kronecker delta.

\section{B. Derivation of the Network Equation}

The electromagnetic fields are expanded as

$$
\boldsymbol{e}=\sum_{n=0} \boldsymbol{e}_{2 n} \boldsymbol{V}_{2 n}, \quad \boldsymbol{a}=\sum_{n=0} \boldsymbol{a}_{2 n+1} \boldsymbol{I}_{2 n+1},
$$

where $\boldsymbol{I}_{2 n+1}=\left(\boldsymbol{I}_{1,2 n+1}, \ldots, \boldsymbol{I}_{M, 2 n+1}\right)^{\mathrm{T}}$ and $\boldsymbol{V}_{2 n}=\left(\boldsymbol{V}_{1,2 n}, \ldots, \boldsymbol{V}_{M, 2 n}\right)^{\mathrm{T}}$ are coefficient vectors. The substitution of (11) into (2) yields

$$
\begin{aligned}
& \boldsymbol{C} \sum_{n=0} \boldsymbol{e}_{2 n} \boldsymbol{V}_{2 n}=-\mathrm{j} \omega \sum_{n=0} \boldsymbol{C} \boldsymbol{a}_{2 n+1} \boldsymbol{I}_{2 n+1} \\
& \boldsymbol{C}^{\mathrm{T}} \boldsymbol{v} \boldsymbol{C} \sum_{n=0} \boldsymbol{a}_{2 n+1} \boldsymbol{I}_{2 n+1}=\boldsymbol{\sigma} \sum_{n=0} \boldsymbol{e}_{2 n} \boldsymbol{V}_{2 n} .
\end{aligned}
$$

From (8) and (12), the electric field is represented by

$$
\sum_{n=0} \boldsymbol{e}_{2 n} \boldsymbol{V}_{2 n}-\boldsymbol{e}_{0} \boldsymbol{V}_{\mathrm{S}}=-\mathrm{j} \omega \sum_{n=0} \boldsymbol{a}_{2 n+1} \boldsymbol{I}_{2 n+1},
$$

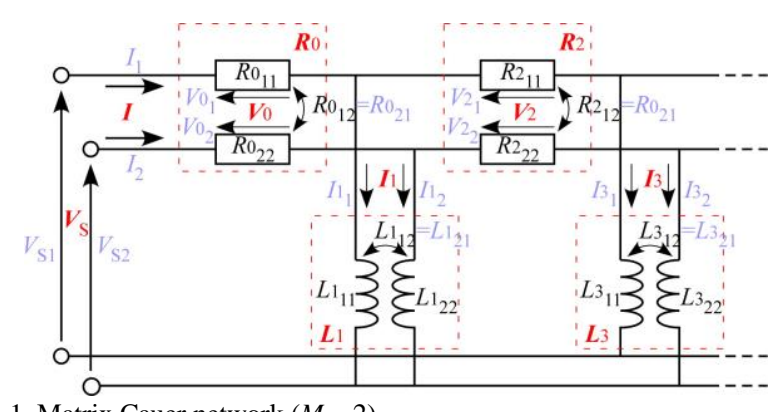

Fig. 1. Matrix Cauer network $(M=2)$.
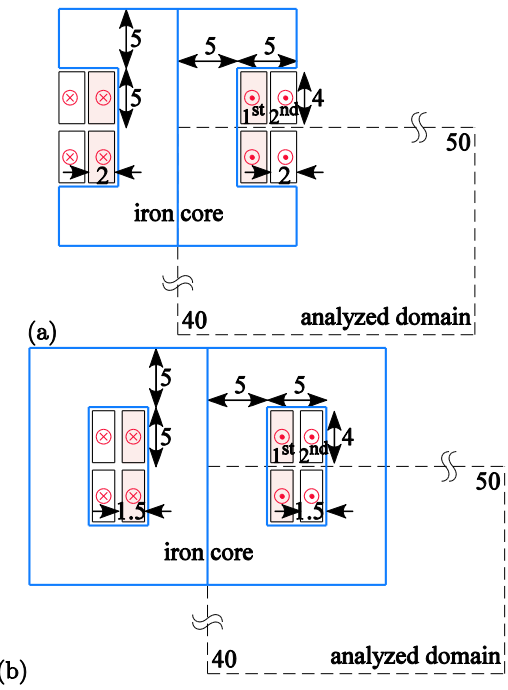

Fig. 2. First and second conductors and iron core (unit: $\mathrm{mm}$ ).

where $\boldsymbol{V}_{\mathrm{S}}=\left(V_{\mathrm{S} 1}, \ldots, V_{\mathrm{S} M}\right)$ is the voltages of power sources.

Multiplying (14) by $\boldsymbol{\sigma} \boldsymbol{e}_{2 k} \boldsymbol{R}_{2 k}$ and using (5) yields

$$
\begin{aligned}
& \boldsymbol{R}_{2 k} \boldsymbol{e}_{2 k}^{\mathrm{T}} \boldsymbol{\sigma}\left(\sum_{n=0} \boldsymbol{e}_{2 n} \boldsymbol{V}_{2 n}-\boldsymbol{e}_{0} \boldsymbol{V}_{\mathrm{S}}\right) \\
& =-\mathrm{j} \omega\left(\boldsymbol{a}_{2 k+1}-\boldsymbol{a}_{2 k-1}\right)^{\mathrm{T}} \boldsymbol{C}^{\mathrm{T}} \boldsymbol{v} \boldsymbol{C} \sum_{n=0} \boldsymbol{a}_{2 n+1} \boldsymbol{I}_{2 n+1}
\end{aligned}
$$

From (10) and (15), it holds that

$$
\begin{aligned}
& \boldsymbol{V}_{0}=\boldsymbol{V}_{\mathrm{S}}-\mathrm{j} \omega \boldsymbol{L}_{1} \boldsymbol{I}_{1} \\
& \boldsymbol{V}_{2 k}=\mathrm{j} \omega\left(\boldsymbol{L}_{2 k-1} \boldsymbol{I}_{2 k-1}-\boldsymbol{L}_{2 k+1} \boldsymbol{I}_{2 k+1}\right) \quad(k=1,2, \ldots)
\end{aligned}
$$

Multiplying (13) by $\boldsymbol{a}_{2 k+1} \boldsymbol{L}_{2 k+1}{ }^{-1}$ and using (6) yields

$$
\begin{aligned}
& \boldsymbol{L}_{2 k+1}{ }^{-1} \boldsymbol{a}_{2 k+1}^{\mathrm{T}} \boldsymbol{C}^{\mathrm{T}} \boldsymbol{v} \boldsymbol{C} \sum_{n=0} \boldsymbol{a}_{2 n+1} \boldsymbol{I}_{2 n+1} \\
& =-\left(\boldsymbol{e}_{2 k+2}-\boldsymbol{e}_{2 k}\right)^{\mathrm{T}} \boldsymbol{\sigma} \sum_{n=0} \boldsymbol{e}_{2 n} \boldsymbol{V}_{2 n} .
\end{aligned}
$$

From (10) and (17), it holds that

$$
\boldsymbol{I}_{2 k+1}=\boldsymbol{R}_{2 k}^{-1} \boldsymbol{V}_{2 k}-\boldsymbol{R}_{2 k+2}{ }^{-1} \boldsymbol{V}_{2 k+2} \quad(k=0,1, \ldots) .
$$

Equations (17) and (18) represent the matrix Cauer circuit, which is shown in Fig. 1 for $M=2$. The impedance matrix is represented by a matrix continued fraction as

$\boldsymbol{Z}=\boldsymbol{R}_{0}+\left\{\frac{1}{\mathrm{j} \omega} \boldsymbol{L}_{1}^{-1}+\left[\boldsymbol{R}_{0}+\left(\frac{1}{\mathrm{j} \omega} \boldsymbol{L}_{3}^{-1}+(\ldots)^{-1}\right)^{-1}\right]^{-1}\right\}^{-1}$

\section{Comparison with the Block Lanczos Method}

The similarity between the single-port CLN and PVL methods discussed in [8] suggests that the multiport CLN method is similar to the block Lanczos method [2]. The multiport CLN method, however, allows the nonorthogonality of basis vectors within a stage ( $\boldsymbol{R}_{2 n}$ and $\boldsymbol{L}_{2 n+1}$ are not diagonal), whereas the block Lanczos method orthogonalizes all the basis vectors. This fact simplifies the CLN procedure by avoiding Gram-Schmidt orthonormalization, and may affect the convergence property, which is examined later. The CLN method is more convenient than the PVL method because the CLN method directly yields the network representation [Fig. 1] whose current and voltages provide the coefficients of the orthogonal expansion of the electromagnetic field (11). Additionally, the multiport CLN does not require the singular value decomposition that is often 
required in other MOR methods. Accordingly, the implementation of multiport CLN is easy because its formulation is as simple as that of the single-port version.

\section{NUMERICAL RESULTS}

First, the admittance matrices with respect to the first and second conductors shown in Fig. 2(a) were computed, where their conductance was $4 \times 10^{7} \mathrm{~S} / \mathrm{m}$ and permeability was $4 \pi \times$ $10^{-7} \mathrm{H} / \mathrm{m}$. Accordingly, the number of ports was two. The bulk-type iron core had permeability of $4 \pi \times 10^{-4} \mathrm{H} / \mathrm{m}$ and conductivity of $1 \times 10^{6} \mathrm{~S} / \mathrm{m}$.

The frequency dependence of admittance components $Y_{11}$ and $Y_{12}$ given by the two-port CLN is shown in Fig. 3, where the CLN was terminated with $\boldsymbol{R}_{2 n}(n=1,2,4,6)$. For comparison, $Y_{11}$ given by both the single-port CLN [8] and block PVL [2] methods is shown in Figs. 4 and 5. The admittances given by FE EC analysis are also shown in Figs. $3-5$. The $2 n$-cycle of the block PVL procedure yielded $2 n$ basis vectors that corresponded to the $n$-cycle of the two-port CLN procedure. The computational cost of EC analysis was roughly evaluated by the number of multiplications of $\boldsymbol{K}^{-1}$ where $\boldsymbol{K}=\boldsymbol{C}^{\mathrm{T}} \mathbf{v} \boldsymbol{C}$. The block PVL method required the operation of $\boldsymbol{K}^{-1}$ once in the first $M(=2)$ steps for the initial setting and twice per cycle of the bi-Lanczos procedure. This means that the $2 n$-cycle of the PVL procedure required $4 n-2$ operations of $\boldsymbol{K}^{-1}$, whereas the two-port CLN required $2 n$ operations of $\boldsymbol{K}^{-1}$. The number of operations of $\boldsymbol{K}^{-1}$ are indicated in parentheses in Figs. 3-5.

The two-port CLN achieved much faster convergence to accurate admittances than the single-port CLN and the multiport CLN proposed in [9] that has the same convergence property as the single-port CLN. The two-port CLN of $n$ cycles with $2 n$ operations of $\boldsymbol{K}^{-1}$ achieved almost the same convergence as the block PVL of $2 n$ cycles with $4 n-2$ operations of $\boldsymbol{K}^{-1}$ since the both schemes use $2 n$ basis vectors. This is expected from the similarities of both methods [14].

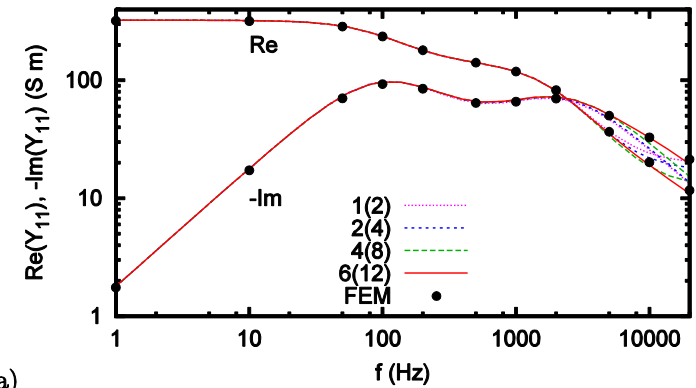

(a)

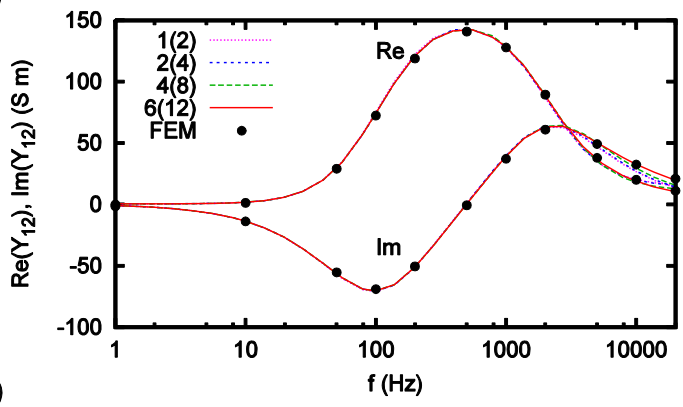

(b)

Fig. 3. Frequency dependence of (a) $Y_{11}$ and (b) $Y_{12}$.

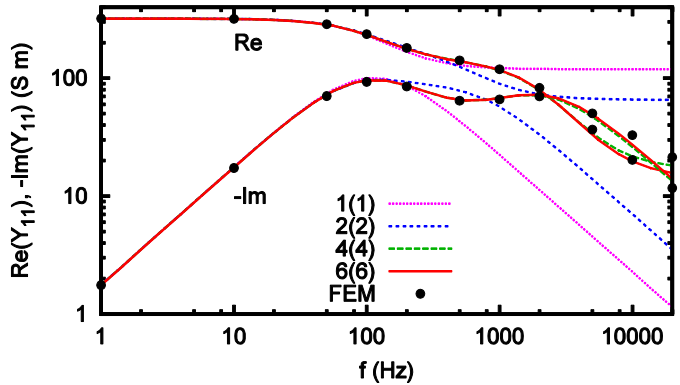

Fig. 4. Frequency dependence of $Y_{11}$ given by the single-port CLN method.

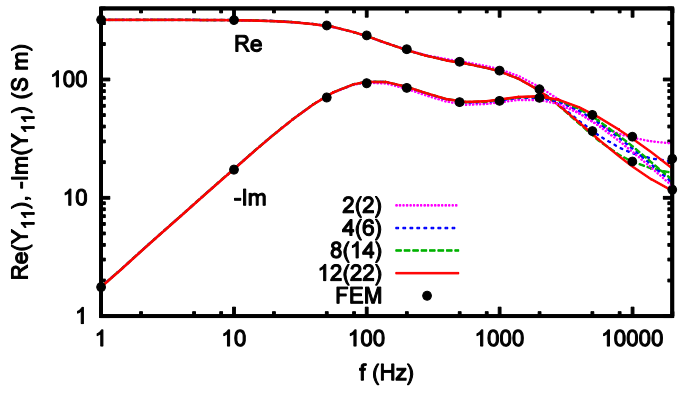

Fig. 5. Frequency dependence of $Y_{11}$ given by the block PVL.

Next, a rectangular voltage waveform of $1 \mathrm{~V}$ [Fig. 6(a)] was fed to the first conductor while the second conductor was short-circuited. The resultant currents $I_{1}$ and $I_{2}$ are shown in Fig. 6, where the multiport CLN [Fig. 6(b)] achieved a more accurate representation than the single-port CLN [Fig. 6(d)]. The magnetic flux distribution at $t=825 \mathrm{msec}$ given by EC FE analysis [Fig. 7(c)] was reconstructed by the two-port CLN [Fig. 7(b)] and single-port CLN [Fig. 7(c)] in 15 stages. The two-port CLN accurately reproduced the flux distribution, whereas the single-port CLN was slightly inaccurate.

Next, the admittance matrices with respect to the first and second conductor shown in Fig. 2(b) were computed by the two-port CLN, single-port CLN, and block PVL methods [Figs. 8, 9], where the material parameters were the same as those in Fig. 2(a). The two-port CLN achieved much faster convergence to accurate admittances than the single-port CLN, and almost the same convergence as the block PVL.

\section{CONCLUSION}

An efficient and convenient multiport MOR method was derived, which directly provided a CLN representation of an EC field without Gram-Schmidt orthonormalization and singular value decomposition. Although the formulation of the multiport CLN is as simple as that of the single-port CLN, its convergence is faster than that of the single-port version and as fast as that of block Lanczos-based MOR. Its practical application to three-phase motor analysis is reported in another work [11].

\section{ACKNOWLEDGMENT}

We thank Maxine Garcia, PhD, from Edanz group (www. edanzediting.com/ac) for editing a draft of this manuscript. 

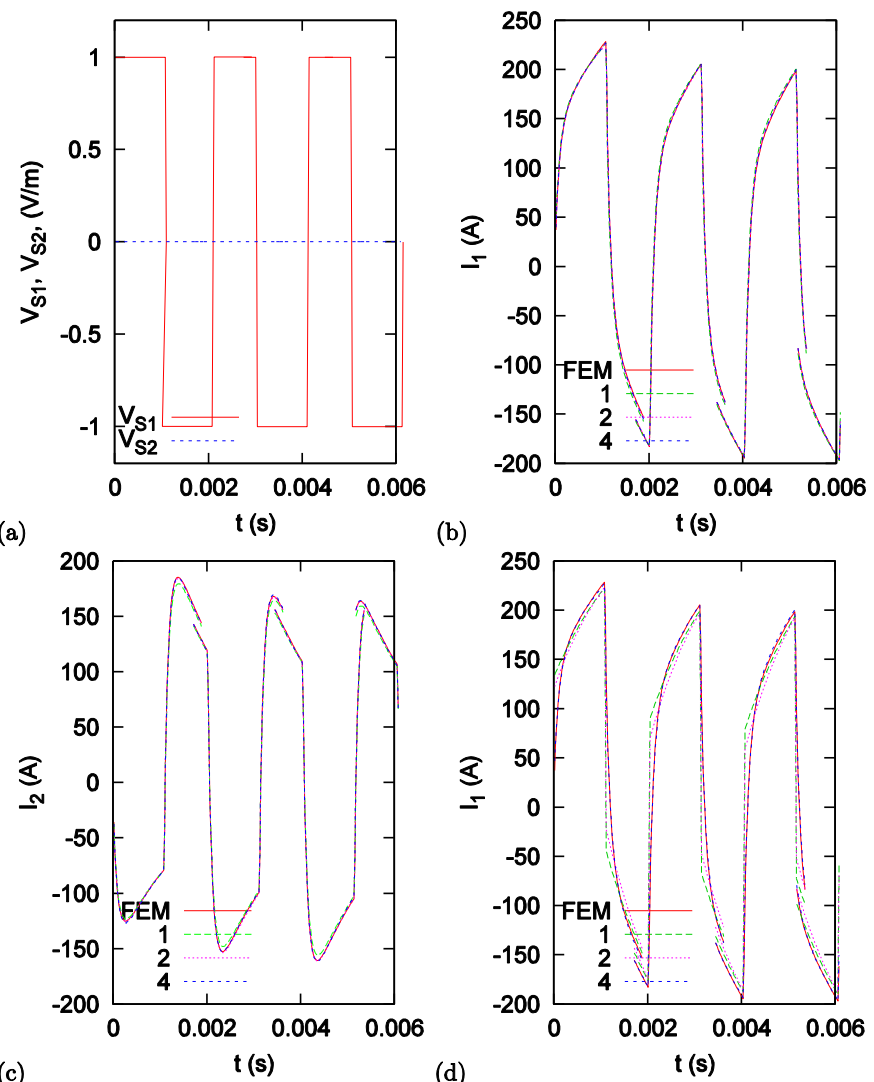

Fig. 6. Time-dependent analysis under rectangular voltage inputs: (a) voltage waveform, and (b) current $I_{1}$, (c) current $I_{2}$, and (d) current $I_{1}$ given by a single-port CLN

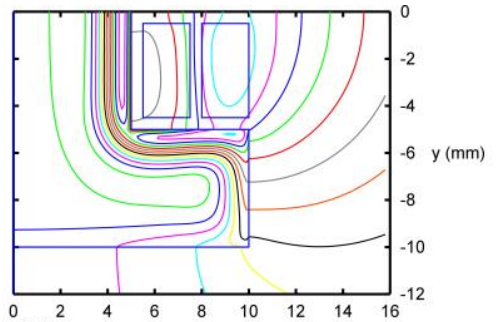

(a)

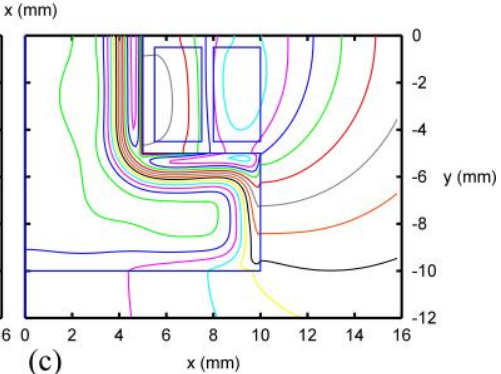

(b)

(c) $x(\mathrm{~mm})$

Fig. 7. Magnetic flux lines given by (a) EC FE analysis, (b) two-port CLN, and (c) single-port CLN.

\section{REFERENCES}

[1] P. Feldmann and R.W. Freund, "Efficient linear circuit analysis by Padé approximation via the Lanczos process," IEEE Trans. Comput.-Aided Design Integr. Circuits Syst., vol. 14, pp. 639-649, May 1995.

[2] P. Feldmann and R. W. Freund, "Reduced-order modeling of large linear subcircuits via a block Lanczos algorithm," Proc. 32nd annual ACM/IEEE Design Automation Conference, pp. 474-479, 1995.

[3] M. Celik and A.C. Cangellaris, "Simulation of dispersive multiconductor transmission lines by Padé approximation via the Lanczos process," IEEE Trans. Microw. Theory Tech., vol. 44, pp. 2525-2535, Dec. 1996.
[4] Y. Sato and H. Igarashi, "Generation of equivalent circuit from finiteelement model using model order reduction," IEEE Trans. Magn., vol. 52, no. 3, 1100304, Mar. 2016.

[5] A. Pierquin, T. Henneron, S. Clénet, S. Brisset, "Model-order reduction of magnetoquasi-static problems based on POD and Arnoldi-based Krylov methods," IEEE Trans. Magn., vol. 51, 7206204, Mar. 2015.

[6] T. Henneron and S. Clénet, "Model-order reduction of multiple-input non-linear systems based on POD and DEI methods," IEEE Trans. Magn., vol. 51, 7207104, Mar. 2015.

[7] A. Kameari, H. Ebrahimi, K. Sugahara, Y. Shindo and T. Matsuo, "Cauer ladder network representation of eddy-current fields for model order reduction using finite element method," IEEE Trans. Magn., vol. 54, 7201804, Mar. 2018.

[8] T. Matsuo, A. Kameari, K. Sugahara, and Y. Shindo, "Matrix formulation of the Cauer ladder network method for efficient eddycurrent analysis," IEEE Trans. Magn., vol. 54, 7205805, Nov. 2018.

[9] K. Sugahara, A. Kameari, T. Matsuo, T. Tokumasu, Y. Shindo, "Multiport analyses in eddy-current fields using principle of superposition of Cauer ladder networks," CEFC Hangzhou, MAP1-11, 2018.

[10] T. Fujiwara, K. Kuriyama, K. Sugahara, A. Kameari, T. Tokumasu, Y. Shindo, and T. Matsuo, "Multi-input MOR method using matrix Cauer form," CEFC Hangzhou, WBP1-3, 2018.

[11] T. Matsuo, K. Sugahara, A. Kameari, Y. Shindo, "Model order reduction of induction motor using Cauer ladder network method, COMPUMAG Paris, OC1-4, 2019.

[12] H. Ebrahimi, K. Sugahara, T. Matsuo, H. Kaimori, A. Kameari, "Modal decomposition of three-dimensional quasi-static Maxwell equations by Cauer ladder network representation," COMPUMAG Paris, PA-A5-2, 2019

[13] Y. Shindo, A. Kameari, K. Sugahara, T. Matsuo, "Dynamical model of an electromagnet using Cauer ladder network representation of eddycurrent fields," IEEJ Trans. IA, vol. 7, pp. 305-313, Apr. 2018

[14] T. Mifune, T. Matsuo, "Relationship between Cauer ladder network representation and CG-like Krylov subspace method," COMPUMAG Paris, PD-M3-1, 2019.
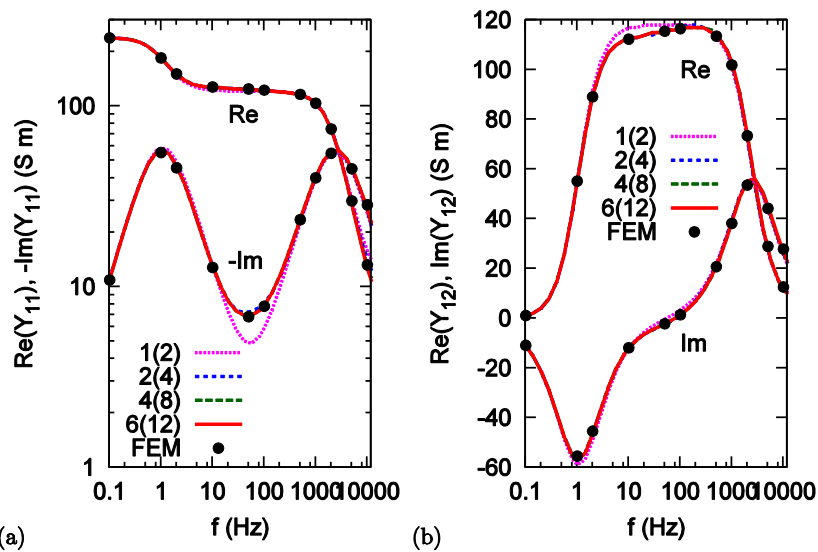

Fig. 8. Frequency dependence of (a) $Y_{11}$ and (b) $Y_{12}$.

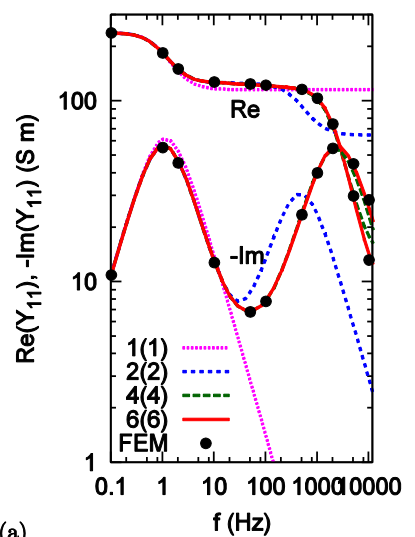

(a)

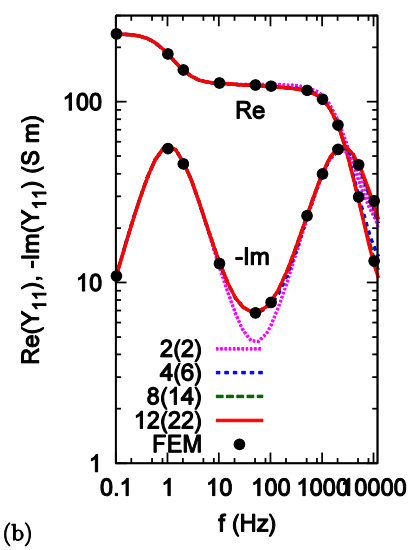

Fig. 9. Frequency dependence of $Y_{11}$ given by the single-port CLN and block PVL methods. 